\title{
Estrés en ganado: causas y consecuencias
}

\author{
Odeón, M.M. ${ }^{1,2}$; Romera, S.A. ${ }^{1,2,3}$ \\ ${ }^{1}$ Instituto de Virología, CICVYA-INTA, Hurlingham, Provincia de Buenos Aires, Argentina. ${ }^{2}$ Consejo Nacional \\ de Investigaciones Científicas y Técnicas-CONICET. ${ }^{3}$ Facultad de Ciencias Exactas, Químicas y Naturales, \\ Univ. de Morón.E-mail: odeon.maria@inta.gob.ar
}

\begin{abstract}
Resumen
Odeón, M.M.; Romera, S.A.: Estrés en ganado: causas y consecuencias. Rev. vet. 28: 1, 69-77, 2017. En los últimos años se está dando una importancia creciente a las normas sobre bienestar animal debido a la confluencia de varios factores, como el mayor conocimiento en distintas disciplinas relacionadas con los animales de producción (el comportamiento animal, la fisiología del estrés y el manejo correcto de los animales), la relación directa entre estos conocimientos y los niveles de producción estables y competitivos a mediano y largo plazo y una mayor concientización social sobre las necesidades de los animales, así como el rechazo hacia los abusos De esta forma, una vez superadas las necesidades de incremento en el abastecimiento de productos, se han empezado a fijar otros parámetros para cumplir con las demandas sociales en el ámbito de la producción ganadera, uno de esos parámetros, cada día más importante, es el bienestar animal. En esta revisión se intenta destacar la importancia del bienestar de los animales, tanto por la calidad de vida de los mismos, como por su impacto sobre la producción ganadera. Se exponen los principales factores generadores de estrés en ganado, así como los efectos fisiológicos y sus impactos negativos sobre la producción. En vías de brindar una mejor vida al animal y aumentar la calidad del producto, cada vez más países y consumidores imponen exigencias legales y reglamentarias que determinan estándares de bienestar para el manejo animal. Es por todo esto que el bienestar animal adquiere cada vez mayor relevancia en todo el mundo. América Latina se encuentra actualmente atravesando un proceso de adaptación a los nuevos requerimientos internacionales.
\end{abstract}

Palabras clave: ganado, bienestar animal, factores de estrés, impacto productivo.

\begin{abstract}
Odeón, M.M.; Romera, S.A.: Stress in productive animals: causes and consequences. Rev. vet. 28: 1, 69-77, 2017. In recent years there has been an increasing importance on animal welfare standards due to the confluence of several factors, including: increased knowledge in different disciplines related to animal production (animal behavior, stress physiology and proper management of animals); the direct relationship between this knowledge and competitive production levels; and greater social awareness of the requirement of animals and the rejection of abuse. Thus, once the needs of increase in the supply of products are met, and other parameters to satisfy social demands in the field of livestock production are set, animal welfare becomes a priority. In this review we try to emphasize the importance of animal welfare, both for the quality of animals' life and its impact on livestock production. The main stressors in cattle as well as the physiological effects and negative impacts on production, are presented. In the process of providing a better life to animals and to increase product quality there is an increasing number of countries with legal and regulatory requirements that determine welfare standards for animal handling. Animal welfare is becoming increasingly important throughout the world and Latin America is currently undergoing a process of adaptation to these international requirements.
\end{abstract}

Key words: livestock, animal welfare, stress factors, productive impact.

\section{OBJETIVO}

En esta revisión se intenta destacar la importancia del bienestar de los animales, tanto por su calidad de vida como por el impacto que promueve sobre la actividad ganadera. Se exponen los principales factores generadores de estrés en el ganado, así como los efectos fisiológicos y sus consecuencias negativas sobre la producción. 


\section{EL ESTRÉS}

La presencia o ausencia de estrés son indicadores potenciales del bienestar animal. A lo largo de la evolución las especies han desarrollado mecanismos fisiológicos y comportamentales para enfrentarse con el estrés, por lo cual únicamente se amenaza la comodidad y vitalidad del ganado cuando se produce un cambio biológico significativo que pone en riesgo el confort y la salud.

Por esta razón, los síntomas del síndrome general de adaptación, lejos de indicar sufrimiento, pueden estar exponiendo que el animal tiene una buena respuesta al medio. Asimismo, las respuestas al estrés no son simples ni constantes sino que dependen de la duración e intensidad del estímulo y de la experiencia de los animales.

En los textos académicos se define al estrés haciendo referencia a un estímulo estresor y a la respuesta que genera ${ }^{29}$. Desde este punto de vista, el estrés se puede definir como una respuesta biológica producida cuando un individuo percibe una amenaza a su homeostasis ${ }^{57}$ . Este término, definido por Walter Cannon, describe un estado estable de parámetros fisiológicos, al que se puede llegar mediante el equilibrio de los mismos dentro de los límites tolerables por diversos mecanismos ${ }^{9}$.

Tal esfuerzo se desarrolla en tres etapas consecutivas denominadas: fase de alarma simpática (breve, fugaz), fase de resistencia (duradera, estrés) y fase de agotamiento (pérdida de la adaptación y ruptura del estado de salud; distrés) ${ }^{10}$. Por lo tanto, resulta importante determinar cuándo el estrés se transforma en distrés y cómo medir ambos.

Las amenazas o estímulos que alejan al individuo de la homeostasises se conocen como agentes estresantes o estresores. Estos últimos se pueden dividir en varios grupos según su naturaleza, duración e intensidad. Según la duración de la respuesta generada, se clasifican en agudos (simples y de duración limitada) o crónicos (duración prolongada) ${ }^{48}$. Un estresor agudo puede pasar a tener una respuesta de estrés crónico si se presenta en forma repetida o cuando su intensidad es anormalmente alta ${ }^{17}$.

Los estresores pueden ser físicos (daño, sobreexigencia, excesivo calor o frío, ruidos) o psicológicos (eventos inesperados, frustración, aislamiento, separación maternal, hechos traumáticos). Todos estos tipos de estresores inducen diferentes respuestas, tanto conductuales como fisiológicas ${ }^{46}$.

La respuesta o defensa biológica contra el agente estresante consiste en alguna combinación de las posibles réplicas generales: la comportamental, la del sistema nervioso autónomo, la neuroendocrina y la inmune. Como resultado se alterará la función biológica, de manera que se producirá un desplazamiento de los recursos lejos de actividades que ocurrían antes de la actuación del estresor, denominándose a este cambio el "costo biológico del estrés". Si éste es prolongado o de gran magnitud, el costo se hace muy elevado y la respuesta supone una carga significativa para el organismo.
Las respuestas conductuales pueden disminuir el riesgo y alejar al individuo del peligro, pero también pueden agravar las consecuencias fisiológicas a través de comportamientos autodestructivos. Esta respuesta incluye alteraciones en los umbrales cognitivos y sensoriales, aumento del estado de alerta, estimulación de la memoria selectiva y supresión de conductas reproductivas ${ }^{51}$.

La respuesta fisiológica (incremento de la presión arterial, aumento de la frecuencia cardíaca, elevación de los niveles de glucocorticoides, alteraciones metabólicas e impedimento de realización de las funciones cognitivas) comienza con la activación del eje hipotálamo-hipófisis-adrenal (HPA), el sistema nervioso autónomo y el sistema inmune (SI), cuyos mediadores fisiológicos son los glucocorticoides (GC), catecolaminas y citoquinas respectivamente ${ }^{21,40}$.

Todos inician eventos celulares que promueven cambios adaptativos en las células y en los tejidos, protegiendo al organismo y promoviendo su supervivencia. En la Figura 1 se muestran las principales estructuras anatómicas involucradas y el funcionamiento del eje HPA.

Generalmente, cuando el estímulo estresante cesa, también lo hace la respuesta que éste desencadenó en el organismo, el cual regresa a su equilibrio original ${ }^{53}$. Por otro lado, si el estresor es muy intenso o de larga duración, lleva al organismo a establecer un nuevo equilibrio, que puede ser beneficioso o perjudicial ${ }^{39,42}$.

El eje HPA es la respuesta neuroendocrina responsable de la regulación de la secreción de GC en la corteza adrenal y de desencadenar las respuestas ante una situación de estrés. Es solamente una parte del gran sistema central que integra las respuestas comportamentales, neuroendocrinas, autonómicas e inmunes frente a alteraciones en la homeostasis ${ }^{12}$.

Una experiencia estresante desencadena la rápida activación del hipotálamo, que estimula al sistema nervioso simpático, el cual inicia la reacción de "lucha o huida" liberando adrenalina y noradrenalina de la médula adrenal, lo que conduce a una respuesta fisiológica. Además, se activa el eje HPA comenzando con la secreción de la hormona liberadora de corticotrofina (CRH) desde el hipotálamo, la cual promueve la liberación de la hormona adrenocorticotropa de la hipófisis, que estimula la liberación de GC en la corteza adrenal.

Los GC se unen a dos tipos de receptores, los de mineralocorticoides y los de glucocorticoides, que se encuentran especialmente en la amígdala, el hipocampo y la corteza prefrontal, además de los órganos periféricos ${ }^{43}$. De esta manera se regula la transcripción y la represión de genes que conducen a cambios a largo plazo y se inhibe el eje HPA por retroalimentación negativa ${ }^{13}$.

Los GC son hormonas esteroideas liberadas por la corteza adrenal. Su principal exponente es el cortisol. Los GC alcanzan todos los órganos a través del sistema circulatorio, estimulan la gluconeogénesis y la glucógenolisis y aumentan la proteólisis y la lipólisis ${ }^{48}$.

Además, movilizan sustratos para el metabolismo energético, para amortiguar la respuesta primaria al es- 


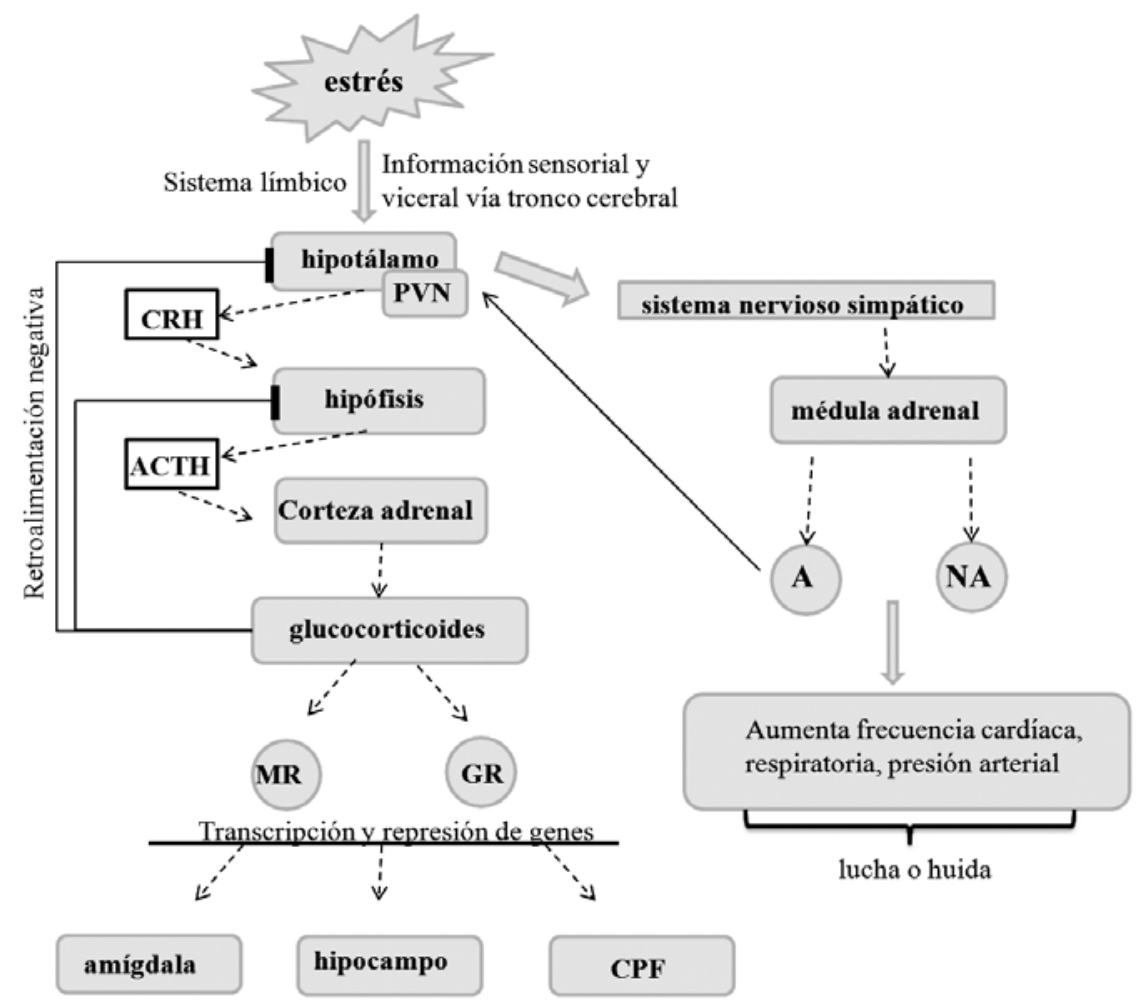

Figura 1. Respuesta al estrés: estructuras anatómicas involucradas en la activación del eje hipotálamo-hipófisisadrenal. A: adrenalina, NA: nor-adrenalina, PVN: núcleo paraventricular, MR: receptor de mineralocorticoides, GR: receptor de glucocorticoides, CPF: corteza prefrontal.

trés y las reacciones inmunes e inflamatorias mediante sus efectos rápidos. El mecanismo subyacente implica una respuesta integrada, que comienza con cambios que llevan a modulaciones más lentas que involucran la transcripción de genes ${ }^{6}$.

Actualmente hay evidencias suficientes que demuestran que las consecuencias negativas que un estímulo aversivo tiene para el animal, no dependen de la naturaleza física de dicho estímulo, sino más bien de la medida en que el animal puede preverlo y controlarlo. Por consiguiente, se ha sugerido que el término "estrés" debería utilizarse sólo cuando la situación a la cual se enfrenta el animal excede la capacidad de regulación del organismo, especialmente cuando dicha situación implica un cierto grado de imprevisibilidad y falta de control ${ }^{41}$.

\section{FACTORES QUE GENERAN ESTRÉS EN EL GANADO}

De manera natural los animales presentan una actitud de alerta y reaccionan ante cualquier estímulo novedoso que observan o escuchan; es un comportamiento innato que le permite sobrevivir en estado libre ${ }^{24}$.

En animales de producción los generadores de estrés pueden ser divididos en factores físicos, sociales (resultantes de las interacciones con los individuos de la misma especie) y las prácticas relacionadas con el manejo. Son estímulos, internos o externos, que activan involuntariamente el eje HPA generando una respuesta al estrés. Su origen es multifactorial, por lo cual sus causas son difíciles de cuantificar, tipificar y por lo tanto controlar.

No existe una definición científica universalmente aceptada de bienestar animal. Es cada vez más amplia, en cambio, la aceptación de criterios generales, como las "cinco libertades" ${ }^{22}$, según las cuales los animales deben ser libres de:

1. hambre, sed y desnutrición

2. miedo y angustia

3. sufrimiento físico y térmico

4. dolor, enfermedad y lesiones

5. manifestar su comportamiento normal

Los principales factores capaces de afectar al ganado son: a) ambientales: cambios de temperatura y humedad relativa, lluvias prolongadas, vientos fuertes, nevadas, sequías, inundaciones; b) régimen de vida: instalaciones precarias, comederos y bebederos de poca capacidad, ambientes sucios, oscuros, ventilación exagerada o deficiente, hacinamiento, promiscuidad; c) manejo: entrenamiento del personal, esquila, destete, transporte, cambios de potrero, arreos largos y apresurados, ordeñe, vacunación; d) nutrición: hambre, sed, sobrealimentación, cambios de nutrición, pasturas suculentas o secas, aguas salobres, alimentos inadecuados para la especie; e) enfermedades: víricas, bacterianas, micóticas, parasitarias, intoxicaciones; y f) factores quirúrgicos y psíquicos: heridas, quemaduras, contusiones, fracturas, operaciones, señalada, sangrías, castigos, gritos, picana eléctrica.

Estos factores estresantes tienen un efecto aditivo, lo cual significa que cuando varios de ellos inciden sobre 
al mismo tiempo, la respuesta de estrés resultante será mayor que si el animal fuese expuesto a un solo factor. Por lo tanto, situaciones como el destete y el transporte pueden ser especialmente difíciles para el ganado ${ }^{15}$.

En muchas especies el estrés dispara un complejo patrón de conducta autonómica similar a lo que sería el temor en los seres humanos. Tanto las experiencias previas como los factores genéticos afectan el temperamento y pueden interactuar en forma compleja para determinar cuánto miedo puede padecer un animal al momento de ser manejado o transportado ${ }^{24}$.

El encierro, la alta densidad animal y el cambio de dieta sumado al destete, favorecen el estrés, así como la presentación y exacerbación de diferentes cuadros o enfermedades. El engorde a corral (feedlot) surgió en la década de 1950 en EEUU ante la necesidad de intensificar la producción, y consiste en encerrar el ganado en rediles donde reciben el alimento en comederos. El período de confinamiento depende del peso inicial y la edad del ganado, variando entre los 60 días y 12 meses. La utilización inadecuada de instalaciones como el piso y la falta de aireación de los corrales a galpón o establos, también generan una respuesta de estrés ${ }^{11}$.

Si bien en nuestro país no es un sistema de producción nuevo, el feedlot ha hecho explosión por razones de mercado a fines de la década de 1990 y en la actualidad hay un nuevo impulso en tal actividad, instalada con firmeza en el sistema productor argentino. Está destinado principalmente a bovinos jóvenes como los provenientes del destete precoz (animales de 90 días) o bien del destete convencional (6 a 10 meses), permaneciendo en engorde hasta su peso de faena, por lo general de 60 a 90 días, hasta llegar a un peso de 230 a 250 kilos en los feedlot productores de "terneros bolitas" o bien de 4 a 6 meses en los feedlot que producen ejemplares de "consumo liviano" (280 a 350 kilos). El mercado exige en estos momentos animales de más de $400 \mathrm{~kg}$, por lo que la estadía en confinamiento se prolonga aún más ${ }^{11}$.

El trabajo de rutina con la hacienda en un sistema extensivo incluye el arreo, la pasada por corrales y manga para prácticas zootécnicas y luego el retorno al pastoreo en el campo. Durante estas tareas, especialmente si el personal no es idóneo, cuando hay apuro o poco control, los animales están expuestos a sufrir los más variados agentes estresores; heridas, a veces fracturas, latigazos, pinchazos y descargas eléctricas, gritos, ladridos, mordeduras, roturas de cola, es decir una amplia gama de agresiones a las que deben adecuar su conducta de adaptación, reacción y resistencia ${ }^{26,30}$.

Aunque el manejo y la inmovilización no sean dolorosos, pueden inducir niveles muy elevados de cortisol, hecho demostrado tanto en ovinos como en bovinos ${ }^{24}$. El nivel de estrés causado por un procedimiento de manejo -como la inmovilización en una manga de compresión- está determinado por la forma en que el animal percibe los estímulos ${ }^{26}$. El estrés causado por un manejo indoloro depende del nivel de miedo.

Un animal criado extensivamente, que ha tenido escaso contacto con personas, tenderá a padecer más estrés por el miedo cuando se lo inmovilice, que un ejemplar criado en contacto estrecho con cuidadores y habituado a los procedimientos de manejo. Se ha demostrado que los terneros criados en contacto estrecho con seres humanos, cuando se los somete a inmovilización ostentan menores niveles de cortisol que quienes mantuvieron menos contacto con el personal ${ }^{25}$.

Un grupo de profesionales norteamericanos estudió los efectos estresantes que ocurren durante las prácticas de rutina en la producción bovina, tales como el destete, el transporte y la vacunación, descubriendo que la causa principal de estrés no fue el transporte sino el manejo y la carga de los animales en un remolque. Sin embargo, la prolongada duración y las deficientes condiciones del transporte produjeron efectos negativos sobre la calidad de la carne ${ }^{5}$.

\section{EFECTOS DEL ESTRÉS SOBRE LA PRODUCCIÓN}

El estrés, además de perturbar el bienestar, afecta negativamente la rentabilidad y viabilidad económica de la actividad ganadera. La respuesta de estrés incluye varias alteraciones que pueden tener consecuencias negativas sobre el rendimiento de los animales de granja. Tales efectos incluyen cambios en la función inmune y consecuente aumento de la susceptibilidad a las enfermedades, la disminución de la ingesta de alimento y de la rumia, afectando la producción, la inhibición de la liberación de oxitocina y la reducción de la fertilidad, entre otros ${ }^{15}$.

Muchos trabajos demuestran que las prácticas operativas y de manejo que aseguran un mayor bienestar animal, logran mejores resultados económicos evitando ineficiencias y pérdidas de valor en toda la cadena de producción ${ }^{22}$. Las repercusiones del estrés impactan profundamente y de manera amplia sobre la productividad, la reproducción y la salud.

La consecuencia del estrés sobre el consumo de alimento ha sido reconocido a pesar de que los cambios precisos involucrados son todavía objeto de debate. Es probable, sin embargo, que el efecto inhibidor del estrés sobre el apetito sea consecuencia de una compleja interacción entre los GC, el CRH y la leptina. También existen evidencias que sugieren un efecto inhibidor sobre la rumia, lo cual reduciría la digestibilidad de los alimentos y consecuentemente el rendimiento productivo, aumentando también el riesgo de acidosis ruminal. El mecanismo preciso del efecto del estrés sobre la rumia no se conoce ${ }^{2}$, aunque está demostrado que cuando el animal supera el límite superior de la zona termo neutral (estrés calórico), el mantenimiento de la temperatura corporal normal empieza a alterar su tasa metabólica basal ${ }^{23}$.

Durante la respuesta de estrés los glucocorticoides contribuyen a aportar la energía necesaria para enfrentar situaciones de amenaza a través de su efecto hiperglucemiante, consecuencia tanto de la glucogenolisis muscular como de la gluconeogénesis hepática ${ }^{59}$. 
El estrés ha demostrado afectar la ganancia de peso y los niveles de producción de derivados industriales. Trabajos científicos indican que el ganado que se observa agitado y nervioso en la manga de compresión, ostenta ganancias de peso significativamente menores, carne más dura, y más cortes lindantes con carne oscura ${ }^{8,58}$.

En Australia se han realizado numerosos estudios que demuestran que cerdas que recibieron un trato agresivo, produjeron menos crías por parición y lograron menores ganancia de peso ${ }^{7,28}$. Los investigadores descubrieron que los porcinos que eran manejados habitualmente a golpes o con picana eléctrica, exhibían ganancias de peso inferiores al promedio. También observaron que la actitud brusca de los operarios y cuidadores afectaba la productividad de las granjas porcinas comerciales $^{28}$, fenómeno que fue también comprobado en vacas lecheras ${ }^{52}$.

En los sistemas tamberos se han comprobado diversos efectos negativos en las etapas de producción, aunque en formas y proporciones diferentes ${ }^{19}$. La vaca lechera es particularmente sensible al clima cálido, el cual le genera disminución del consumo de alimento, afectando el nivel de producción. Para estos animales, la principal estrategia para aminorar el calor corporal es la rebaja voluntaria del consumo de materia seca. Por ello, la merma en la ingesta de alimento llega hasta un $25 \%$ con la consecuente reducción de la producción de leche.

Datos de Israel reportan diferencias de hasta 1.000 $\mathrm{kg}$ por lactancia entre las vacas paridas en verano y en invierno, así como una pérdida promedio anual de 350 $\mathrm{kg}$ de leche durante el verano ${ }^{23}$. Además de la reducción en la producción de leche, los efectos fisiológicos también se aprecian en el retraso del crecimiento de los animales de reemplazo, ocasionando pérdidas importantes al productor.

Cabe mencionar que en muchas regiones de nuestro país, incluso en numerosos establecimientos de la pampa húmeda, durante varios meses del año las vacas sufren el llamado "hambre a fecha fija". Es algo que, en las zonas marginales (La Rioja, Catamarca, San Juan, Mendoza), llega a su máxima expresión, proyectándose también a buena parte de la Patagonia árida. En estos animales se producen cambios intracelulares muy notables, llegando a una acidosis metabólica compensada, compatible con la vida, pero que se traduce en una productividad muy disminuida ${ }^{18,30}$.

En las plantas de faena, el manejo apacible de los cerdos contribuye a mantener la calidad de la carne. El trato rudo, los amontonamientos y el uso excesivo de la picana eléctrica antes del noqueo, aumentan la carne pálida, suave y exudante, efectos no deseados ${ }^{25}$. Las consecuencias del manejo sobre el bienestar animal se pueden medir a través de indicadores fisiológicos y de comportamiento, en tanto los efectos cuantitativos y cualitativos sobre la producción de carne se valoran en general a través de los cambios de peso vivo y de la canal, los daños en las canales (contusiones) y las alteraciones de $\mathrm{pH}$ y color en la carne ${ }^{54}$.
Se afirma que el estrés provoca cambios medibles post mortem a nivel muscular (en canal o carne), los que están fundamentalmente relacionados con los niveles de glucógeno, el pH y el color ${ }^{20}$. En el caso de los bovinos la principal influencia del manejo ante mortem sobre la calidad de la carne es a través de su efecto potencial sobre las reservas de glucógeno muscular; si éstas se agotan el nivel de acidificación post mortem se reduce, conduciendo al llamado "corte oscuro" 59 .

La carne resultante tiene $\mathrm{pH}$ alto, generalmente sobre 6,0, apareciendo seca, firme y oscura (DFD = dark, firm, dry) ${ }^{32}$. El pH tiene gran importancia en cuanto a las características organolépticas de la carne y sobre su aptitud para transformarse en productos procesados, ya que influye sobre ciertos parámetros de calidad como la terneza, el color y la capacidad de retención de agua ${ }^{27}$. Un pH final elevado aumenta la incidencia de carnes DFD, las cuales ostentan mayor riesgo de contaminación bacteriana ${ }^{55}$.

Adecuadas cantidades de glucógeno muscular resultan en óptimos valores de $\mathrm{pH}$ final, cuyo nivel puede variar por diversos factores como raza ${ }^{35}$, estado nutricional ${ }^{3}$, temperamento ${ }^{36} \mathrm{y}$ estrés pre-faena. De este modo, diversos factores de estrés son capaces de alterar las reservas musculares de glucógeno, y por lo tanto, afectar el $\mathrm{pH}$ final, resultando en diversos defectos de la calidad de la carne obtenida ${ }^{4}$.

En cuanto al transporte y el ayuno, los estudios realizados han demostrado que tiempos de transporte terrestre de 16 o más horas y esperas prolongadas sin alimento en el matadero (más de 12 horas), afectan negativamente el bienestar animal y la calidad de la carne del bovino ${ }^{56}$. Básicamente hay una tendencia a perder peso de la canal, hay más contusiones, más alteraciones de $\mathrm{pH}$ y de color en la carne, impactando sobre la vida útil de la misma y afectando procesos tecnológicos tan importantes como el envasado al vacío, uno de los principales problemas de calidad en las plantas faenadoras ${ }^{62}$.

También se ha demostrado que el estrés agudo prefaena tiene un fuerte impacto negativo sobre la calidad de la carne. Se constató que puede afectar el grado de proteólisis ocurrida durante la maduración llevada a cabo durante 21 días. Dado que dicho efecto fue observado en animales provenientes de sistemas de producción contrastantes (intensivo y extensivo), ello sugiere que los mecanismos operantes serían independientes de las variables relacionadas con el sistema que haya originado dichos cortes ${ }^{49}$.

Estas características constituyen una limitante de calidad para la exportación, ya que son parámetros que se detectan recién después de haber cumplido todos los demás requisitos sanitarios, de trazabilidad y de composición requeridos por cada cliente. El problema del corte oscuro se puede prevenir en gran medida con programas que contemplen el trabajo bajo normas de bienestar animal en el manejo previo, como un transporte directo del predio al matadero ${ }^{49,50}$. 


\section{EFECTOS DEL ESTRÉS SOBRE LA REPRODUCCIÓN}

Evolutivamente, la inhibición de la reproducción en situaciones estresantes parece tener ventajas que le evitan al animal riesgos en épocas de alta demanda metabólica. La activación del eje HPA como respuesta al estrés afecta de modo significativo el funcionamiento reproductivo de varias especies. Prácticas como el transporte, el aislamiento y la inmovilización activan dicho eje y pueden inhibir la secreción de la hormona liberadora de gonadotrofina desde el hipotálamo, de la hormona luteinizante desde la hipófisis y la producción de estradiol folicular.

En conjunto, ello conduce a que situaciones de estrés interfieran con la eficiencia reproductiva, incrementando el número de servicios por concepción y aumentando la proporción de animales en los que la conducta de estro no se manifiesta ${ }^{2}$. Ejemplos concretos de este fenómeno se han demostrado científicamente, como la utilización de picanas eléctricas o la inmovilización, que debilitan el funcionamiento reproductivo de las hembras de distintas especies ${ }^{25,31}$.

Los efectos del estrés calórico en la reproducción bovina han sido estudiados ampliamente. Sus consecuencias adversas se han reportado en aspectos como la expresión y duración del estro, el desarrollo embrionario temprano, el flujo sanguíneo, los niveles hormonales y el crecimiento fetal ${ }^{38}$.

El ciclo estral es un evento fisiológico sensible al estrés, el cual disminuye la intensidad y duración del celo, ejerciendo efectos nocivos sobre la fertilidad ${ }^{48}$ . Las altas temperaturas ambientales también afectan la fertilidad, el comportamiento sexual, la folículo-génesis, la ovulación, la función luteal y la implantación. Estos problemas operan negativamente sobre la tasa de natalidad, incrementando el intervalo entre partos ${ }^{44}$.

El estrés también se ha asociado con el aumento de la cantidad de óvulos no fertilizados y de embriones anormales. En hatos afectados por el calor se observa falta de concepción, muertes embrionarias tardías e incluso abortos ${ }^{25}$. Los efectos más conocidos sobre la gestación son: menor peso del ternero al nacimiento, alteraciones en las concentraciones hormonales maternofetales y reducción en la producción de leche posparto.

El estrés causa un descenso considerable en el flujo sanguíneo uterino, el cual está asociado con la disminución en el crecimiento fetal durante la gestación tardía y alteración de la funcionalidad de la placenta y de la actividad endocrina. El resultado es un ternero de menor peso al nacer y alteraciones que afectan el desarrollo mamario y la producción de leche.

\section{EFECTOS DEL ESTRÉS SOBRE LA SALUD}

Se sabe que tanto en porcinos como en bovinos, el estrés del transporte o de la inmovilización disminuye la función inmunitaria ${ }^{14,45,58}$. Los animales que sufren confinamiento o estrés social (alejamiento, abandono, aislamiento) muestran una actividad inmunitaria deprimida. Investigaciones realizadas en seres humanos y en animales de experimentación han revelado que el estrés puede afectar la respuesta inmune tanto humoral como celular.

El eje HPA regula al SI principalmente por la liberación de GC. Los GC ejercen una variedad de efectos a través de mecanismos de señalización de hormonas esteroideas. Las células del SI poseen receptores para GC, los cuales pueden ejercer su acción regulatoria sobre el SI por varias vías ${ }^{37}$. Modulan la expresión de citoquinas, moléculas de adhesión, quimioatractantes y otros factores inflamatorios ${ }^{1}$, y bloquean la maduración linfocitaria ${ }^{63}$.

Durante muchos años se pensó que los GC eran inmunosupresores, pero los estudios que llevaron a estas conclusiones se realizaron utilizando moléculas sintéticas y dosis farmacológicas. Es importante reconocer que los GC en dosis farmacológicas ejercen funciones diferentes a las fisiológicas. En concentraciones fisiológicas resultan en una modulación de la respuesta inflamatoria, mientras que en las dosis farmacológicas, mucho mayores que las fisiológicas, resultan en una supresión total de la respuesta inflamatoria ${ }^{60}$.

Por lo tanto, hay que tener presente que los efectos de los GC administrados no son los mismos que los producidos por los GC endógenos y el estrés. Los mecanismos responsables del efecto del estrés crónico sobre el sistema inmune son muy específicos y afectan solamente algunos tipos de defensa. En la práctica, esto significa que algunas patologías son más susceptibles de ser potenciadas por el estrés crónico que otras. Entre dichas patologías se incluyen las enfermedades respiratorias infecciosas y la contaminación por Salmonella $s p^{2}$.

Se ha demostrado que el estrés del transporte aumenta la incidencia de neumonía causada por el herpes virus bovino tipo-1 en terneros, la neumonía causada por Pasteurella $s p$ y la consiguiente mortalidad en terneros y ovinos, así como la samonelosis en ovinos y equinos ${ }^{2}$. La susceptibilidad a otras enfermedades también puede aumentar como consecuencia de situaciones potencialmente estresantes.

Por ejemplo, varios estudios han demostrado un aumento en la prevalencia de mastitis en vacas lecheras como resultado del miedo crónico. Aunque no se conoce el mecanismo preciso que explica este efecto, se ha sugerido que la función de las células "natural killer" podría verse afectada por el estrés y esto a su vez podría conducir a un aumento de la susceptibilidad de la glándula mamaria a los agentes infecciosos ${ }^{2}$.

Es oportuno mencionar que el estrés interviene muy directamente en la absorción de los medicamentos, aumentando su toxicidad y provocando que muchos tratamientos, bajo condiciones estresantes, resulten ineficaces o tóxicos.

Ha sido ampliamente documentado el impacto negativo que el estrés tiene sobre el sistema inmune ${ }^{17}$. Los mecanismos moleculares por los cuales el estrés induce el desarrollo de patologías están en continuo 
estudio, pero poco se sabe del impacto que tienen a nivel de poblaciones celulares activadas y del perfil de citoquinas involucradas. Se han documentado algunos efectos puntuales, como por ejemplo, en animales estresados es común observar neutrofilia, la cual puede ocurrir por liberación de médula ósea causada por glucocorticoides ${ }^{34}$.

También se ha reportado que el estrés por transporte incrementa la fagocitosis oxidativa y la expresión de moléculas de adhesión de neutrófilos de bovinos ${ }^{33}$. El "leucograma de estrés" es la disminución de linfocitos y eosinófilos, generalmente acompañada del aumento de leucocitos totales y neutrófilos.

El estado sanitario tiene un impacto económico significativo. Los factores que contribuyen a la aparición de enfermedades deben tenerse en cuenta para evitar mayores pérdidas económicas y garantizar el bienestar de los animales.

\section{CONCLUSIONES}

Se ha destacado la importancia del bienestar de los animales, tanto por su calidad de vida como por el impacto sobre la producción ganadera. Se expusieron los principales factores generadores de estrés en ganado, así como los efectos fisiológicos y sus impactos negativos sobre la producción.

El conocimiento de la conducta y temperamento de los animales, así como de los factores que desencadenan su reactividad, son esenciales para sistematizar el manejo del personal y para mejorar el diseño de las instalaciones. El estrés afecta negativamente los procesos de obtención de carne tanto en los rendimientos físicos como en la calidad del producto.

El bienestar animal puede conducir a alimentos más seguros y de mayor calidad, así como al aumento de la competitividad de los productos del sector. Tales conocimientos deben estar basadas en datos científicos confiables. Cuanto más complejos son los temas, la necesidad de evidencia objetiva aumenta. Los niveles plasmáticos de glucocorticoides y los cambios conductuales se han utilizado como medidas de estrés, pero es necesario ampliar la búsqueda de biomarcadores para así poder controlar y monitorear este estado, tanto en los sistemas de producción como en investigación.

En el área científica también son importantes el bienestar y el control del estado de los animales, ya que garantizan que las investigaciones arrojen resultados más confiables y robustos. Esto a su vez, proporciona el fundamento científico para trasladar el conocimiento al sector productivo y la extrapolación de resultados en el campo de la medicina, donde también se utilizan animales de granja como modelos experimentales.

Se debe alentar al sector cárnico internacional para que adopte buenas prácticas de manejo y de bienestar. Esto es especialmente importante en los países en desarrollo, ya que mejoraría su producción. En estas naciones se pueden implementar buenas normas de bienestar animal sin el uso de equipos costosos de alta tecnolo- gía. Se puede recibir asistencia para realizar, local o regionalmente, mejoramientos sencillos. Se requiere con urgencia la implementación de mejores prácticas y métodos de cría, manejo, transporte y sacrificio, así como un marco legal que los contenga.

Con este propósito existe bibliografía con recomendaciones, regulaciones y normas que se sugiere consultar. Hay guías disponibles tanto en Estados Unidos como en la Unión Europea, tales como la "Guide for the care and use of agricultural animals in research and teaching" (2010) ${ }^{16}$, la "Directiva europea" (2010) ${ }^{47}$ y los "First european protocols for assessing farm animal welfare" (2009) ${ }^{61}$.

Actualmente se conocen varias herramientas de las que disponen los productores para mejorar su cometido: buenas prácticas de bienestar animal, manejo sanitario y preventivo contra enfermedades, evaluación de la capacidad reproductiva y manejo eficiente de la alimentación, por citar sólo algunos de ellos. Muchos países y consumidores imponen exigencias reglamentarias y/o legales que determinan estándares de bienestar con los que se deben manejar los animales para poder comercializar sus derivados, brindando una mejor vida al animal y aumentando la calidad del producto.

El bienestar animal adquiere cada vez mayor relevancia en todo el mundo. América Latina se encuentra actualmente atravesando un proceso de adaptación a los nuevos requerimientos internacionales, para mantenerse dentro del mercado mundial. Si bien en Argentina es un tema que se ha instalado desde hace algunos años, es necesario ampliar el conocimiento y las investigaciones sobre los mecanismos de estrés en sistemas de producción, transporte y faena, así como actualizar la reglamentación pertinente.

\section{REFERENCIAS}

1. Adcock IM, Ito K. 2000. Molecular mechanisms of corticosteroid actions. Monaldi Arch Chest Dis 55: 256-266.

2. Alvarez L. 2008. Efectos negativos del estrés sobre la reproducción en animales domésticos. Arch Zoot 57: 39-59.

3. Andersen SL, Teicher MH. 2008. Stress, sensitive periods and maturational events in adolescent depression. Trends Neurosci 31: 183-191.

4. Apple JK. 1995. Effects of restraint and isolation stress and epidural blockade on endocrine and blood metabolite status, muscle glycogen metabolism, and incidence of dark-cutting longissimus muscle of sheep. J Anim Sci 73: 2295-2307.

5. Avant S. 2013. Temperament plays key role in cattle health. Agric Res 61: 20.

6. Baker M, Funder JW, Kattoula SR. 2013. Evolution of hormone selectivity in glucocorticoid and mineralocorticoid receptors. J Steroid Biochem Mol Biol 137: 57-70.

7. Barnett JL, Edge MK, Hemsworth PH. 2008. The place of quality assurance in managing animal welfare during long distance transport. Vet Ital 44: 121-131.

8. Blokhuisa HJ, Keelinga A, Gavinellib JS. 2008. Animal welfare's impact on the food chain. Trends Food Sci \& Technol 19: 579-587. 
9. Cannon W. 1935. Stresses and strains of homeostasis. Am J Med Sci 189: 13-14. http://journals.lww.com/amjmedsci/ toc/1935/01000 (December 5, 2014)

10. Coppo JA. 2001. ¿Estrés o alarma simpática? Actualización bioquímico-clínica. Selec Vet 9: 336-342.

11. Costa F. 2005. El manejo del estrés y la salud en los sistemas intensivos. XVI Jorn Ganad Pergamino, www.produccion-animal.com.ar/sanidad_intoxicaciones.../31-stres.pdf.

12. Dallman MF, Bhatnagar S, Viau V. 2006. Hypothalamic-pituitary-adrenal axis. In: Encyclopedia of stress, $2^{\circ}$ ed, Elsevier, London, p. 468-476.

13. Deppermann S, Storchak H, Fallgatter AJ, Ehlis, AC. 2014. Stress-induced neuroplasticity: (mal)adaptation to adverse life events in patients with PTSD. A critical overview. NeuroSci 26: 166-177.

14. Edwards TA. 2010. Control methods for bovine respiratory disease for feedlot cattle. Food Anim Pract 2: 273-284.

15. Farm Animal Welfare Education. 2013. Estrés en animales de granja: concepto y efectos sobre la producción. www.innocua.net/web/download-1117/vi-welfare-factsheet-es.pdf

16. FASS Board. 2010. Guide for the care and use of agricultural animals in research and teaching. http://www.fass. org/docs/agguide3rd/Ag_Guide_3rd_ed.pdf

17. Fink G. 2000. Encyclopedia of stress, 2nd ed., Academic Press, New York, 2300 p.

18. Fité EG. 2008. Caracterización de la ganadería bovina en Patagonia Sur. http://www.produccion-animal.com.ar/ informacion_tecnica/origenes_evolucion y e-stadisticas_ de_la_ganaderia/103-Patagonia_Sur.pdf

19. Flamenbaum I, Gallon, IN. 2010. Management of heat stress to improve fertility in dairy cows in Israel. $J$ Reprod Dev 56: 36-41.

20. Forrest JC. 1979. Fundamentos de ciencia de la carne, Ed. Acribia, Zaragoza, España, 361 p.

21. Foster AC, Gill R, Kemp JA, Woodruff GN. 1987. Systemic administration of MK-801 prevents N-methyl-D-aspartate-induced neuronal degeneration in rat brain. Neurosci letters 76: 307-311.

22. Giménez ZM. 2006. El bienestar animal y la calidad de la carne: buenas prácticas de manejo del ganado. w.produccion-animal.com.ar/etologia_y_bienestar/bienestar_en_general/42-punto_clave.pdf

23. González PJ. 2011. El estrés calórico en los bovinos. www.medigraphic.com/pd fs/abanico/av-2014/av143d.pdf

24. Grandin T. 1997. Evaluación del estrés durante el manejo y transporte (traducción Dr. Marcos Giménez-Zapiola). $J$ Anim Sci 75: 249-257.

25. Grandin T. 1998. La reducción del estrés del manejo mejora la productividad y el bienestar animal. http://www. grandin.com/spanish/reduccion.estres.manejo.html

26. Grandin T, Shivley C. 2015. How farm animals react and perceive stressful situations such as handling, restraint, and transport. Animals 5: 1233-1251.

27. Gregory NG. 2008. Animal welfare at markets and during transport and slaughter. Meat Sci 80: 2-11.

28. Hemsworth PH, Barnett GL, Coleman GJ, Hansen C. 1989. A study of the relationships between the attitudinal and behavioral profiles of stockpersons and the level of fear of humans and reproductive performance of commercial pigs. Appl Anim Behav Sci 23: 301-314.

29. Herlenius E, Lagercrantz H. 2004. Development of neurotransmitter systems during critical periods. Exp Neurol 190: S8-S21.

30. Hinsch O. 1974. El estrés en el ganado. Dinámica Rural 67: 23-27.

31. Hixon DL, Kesler DK, Troxel TR. 1981. Reproductive hormone secretions and first service conception rate subsequent to ovulation control with Synchromate B. Theriogenol 16: 219-229.

32. Hood D, Tarrant PV. 1980. The problem of dark-cutting in beef. In: Current Topics in Vet Med Anim Sci 10: 141-164.

33. Hulbert LE. 2011. Innate immune responses of temperamental and calm cattle after transportation. Vet Immunol Immunopathol 143: 66-74.

34. Kelley KW. 1981. Whole blood leukocyte vs. separated mononuclear cell blastogenesis in calves: time-dependent changes after shipping. Can J Comp Med 45: 249-258.

35. King DA, Morgan WW. 2006. Carcass merit between and among family groups of Bos indicus crossbred steers and heifers. Meat Sci 72: 496-502.

36. King DA et al. 2006. Influence of animal temperament and stress responsiveness on the carcass quality and beef tenderness of feedlot cattle. Meat Sci 74: 546-556.

37. Lasselin J, Alvarez E, Grigoleit JS. 2016. Well-being and immune response: a multi-system perspective. Current Opin Pharmacol 29: 34-41.

38. López-Gatius F. 2012. Factors of a noninfectious nature affecting fertility after artificial insemination in lactating dairy cows. Theriogenol 1(77): 1029-1041.

39. McEwen BS. 1998. Stress, adaptation, and disease. Allostasis and allostatic load. Annals New York Acad Sci 840: 33-44.

40. McEwen BS. 2002. Sex, stress and the hippocampus: allostasis, allostatic load and the aging process. Neurobiol Aging 23: 921-939.

41. McEwen BS. 2005. Stressed or stressed out: What is the difference? J Psychiatry NeuroSci 30: 315-318.

42. McEwen BS. 2006. Definitions and concepts of stress. In: Encyclopedia of stress (Fink G, ed.), vol. 3, Academic Press, San Diego, p. 508-515.

43. McEwen BS. 2015. Mechanisms of stress in the brain. Nat Neurosci 18: 1353-1363.

44. Mellado M. 2014. Effects of month of breeding on reproductive efficiency of Holstein cows and heifers inseminated with sex-sorted or conventional semen in a hot environment. Trop Anim Health \& Prod 46: 265-269.

45. Mertshing HJ, Kelly AW. 1983. Restraint reduces the size of thymus gland and pha swelling in pigs. J Anim Sci 57: 175.

46. Myers B, Scheimann J, Franco A, Herman JP. 2016. Ascending mechanisms of stress integration. https://www. researchgate.net/publication/303358516.

47. Official Journal of the European Union: Directive 2010/63/eu of the European Parliament on the Protection of Animals Used for Scientific Purposes. ec.europa.eu/.../ lab_animals/legislation_en.htm 
48. Pacak K. 2006. Acute stress response. In: Experimental encyclopedia of stress (Fink G, ed.), $2^{\circ}$ ed., Elsevier, Amsterdam, p. 8-16.

49. Pighin D. 2014. Influencia del estrés animal pre-faena sobre parámetros de calidad de carne fresca y madurada. http://inta.gob.ar/documentos/influencia-del-estres-animal-pre-faena-sobre-parametros-de-calidad-de-carnefresca-y-madurada.

50. Pighin D et al. 2016. A contribution of beef to human health: a review of the role of the animal production systems. Sci World J article 8681491.

51. Pines G. 1992. Cloning and expression of a rat brain Lglutamate transporter. Nature 360: 464-467.

52. Seabrook MF. 1972. A study to determine the influence of the herdsman's personality on milk yield. J Agric Labor Sci 1: 45-59.

53. Selye H. 1946. The general adaptation syndrome and the diseases of adaptation. J Clin Endocrinol Metab 6: 117-230.

54. Sepúlveda N, Gallo C, Allende R. 2007. Importancia del bienestar animal en producción bovina. Arch Latinoam Prod Anim 15: 127-132.

55. Silva JA, Patarata L, Martins C. 1999. Influence of ultimate $\mathrm{pH}$ on bovine meat tenderness during ageing. Meat Sci 52: 453-459.

56. Tadich N, Gallo MV, Alvarado M. 2000. Efectos de 36 horas de transporte terrestre con y sin descanso so- bre algunas variables sanguíneas indicadoras de estrés en bovinos. $w w w . s c i e l o . c l / s c i e l o . p h p ?$ script $=s c i$ arttext...732X2000000200004.

57. Trotti DS, Nussberger AV, Hediger MA. 1997. Differential modulation of the uptake currents by redox interconversion of cysteine residues in the human neuronal glutamate transporter EAAC1. Europ J Neurosci 9: 2207-2212.

58. Voisinet BD, Grandin T, Tatum JD, O'Connor SF, Struthers JJ. 1997. Feedlot cattle with calm temperaments have higher average daily weight gains than cattle with excitable temperaments. J Anim Sci 75: 892-896.

59. Warriss PD. 2010. Post-mortem changes in muscle and its conversion into meat. In: Meat Science: An introductory text, Cab International, Wallingford, UK, p. 65-76.

60. Webster JI, Tonelli L, Sternberg EM. 2002. Neuroendocrine regulation of immunity. Ann Rev Immunol 20: 125-163.

61. Welfare Quality. 2009. First European Protocols for assessing farm animal welfare. www.welfarequality.net/everyone/43148/9/0/22

62. Wirth F. 1987. Tecnología para la transformación de carne de calidad anormal. Fleischwirtsch 1: 22-28.

63. Wisniewska M, Pyrzynska B, Kaminska B. 2004. Impaired AP-1 dimers and Nfat complex formation in immature thymocytes during in vivo glucocorticoid-induced apoptosis. Cell Biol Int 28: 773-780.

\section{Asociación Cooperadora de la Facultad de Ciencias Veterinarias Universidad Nacional del Nordeste}

Personería Jurídica No 647/92 y 912/00

Sargento Cabral 2139

3400 Corrientes

Argentina

La Asociación Cooperadora de la Facultad de Ciencias Veterinarias de la UNNE fue constituida el 10 de diciembre del año 1991 como entidad de bien público, con el objeto de promover y coadyuvar las actividades científicas, educativas y culturales relacionadas con las Ciencias Veterinarias. En tal sentido, implementa acciones para colaborar con la enseñanza, extensión, actualización y difusión científica que realiza dicha casa de estudios.

\section{Beneficios que brinda a sus asociados:}

- Fotocopias con descuentos especiales en la Fotocopiadora Copias.com que funciona dentro del predio de la Facultad de Ciencias Veterinarias.

- Descuentos para la adquisición de libros de distintas editoriales.

- Descuentos especiales en otros rubros.

Tel. (0379) 4425753 interno 186•Página Web www.vet.unne.edu.ar•E-mail: cooperadora@vet.unne.edu.ar 